\title{
Intratumoral and Peritumoral Apparent Diffusion Coefficient and MGMT mRNA Expression in Different Meningioma Histopathological Grade
}

\author{
Rahmad Mulyadi ${ }^{1, *}$, Mochammad Hatta ${ }^{2}$, Andi Asadul Islam ${ }^{3}$, Bachtiar Murtala ${ }^{4}$, \\ Jumraini Tammase ${ }^{5}$, Muhammad Firdaus ${ }^{6}$, Eka Susanto ${ }^{7}$, Joedo Prihartono ${ }^{8}$ \\ ${ }^{1}$ Neuroradiology Division, Department of Radiology, Faculty of Medicine, Universitas Indonesia/RSUPN Dr. Cipto Mangunkusumo, \\ J1. Diponegoro No. 71, Jakarta 10430, Indonesia \\ ${ }^{2}$ Molecular Biology and Immunology Laboratory, Faculty of Medicine, Universitas Hasanuddin, J1. Perintis Kemerdekaan KM 10, \\ Makassar 90245, Indonesia \\ ${ }^{3}$ Department of Neurosurgery, Faculty of Medicine, Universitas Hasanuddin, Jl. Perintis Kemerdekaan KM 10, Makassar 90245, Indonesia \\ ${ }^{4}$ Department of Radiology, Faculty of Medicine, Universitas Hasanuddin, Jl. Perintis Kemerdekaan KM 10, Makassar 90245, Indonesia \\ ${ }^{5}$ Department of Neurology, Faculty of Medicine, Universitas Hasanuddin, Jl. Perintis Kemerdekaan KM 10, Makassar 90245, Indonesia \\ ${ }^{6}$ Department of Neurosurgery, Dharmais Hospital National Cancer Center, Jl. Letjen Jend. S. Parman No.84-86, Jakarta 11420, Indonesia \\ ${ }^{7}$ Department of Anatomical Pathology, Faculty of Medicine, Universitas Indonesia/RSUPN Dr. Cipto Mangunkusumo, J1. Diponegoro No. 71, \\ Jakarta 10430, Indonesia \\ ${ }^{8}$ Department of Community Medicine, Faculty of Medicine, Universitas Indonesia/RSUPN Dr. Cipto Mangunkusumo, Jl. Diponegoro No. 71, \\ Jakarta 10430, Indonesia \\ *Corresponding author. E-mail: dr_rahmad_radiologi@yahoo.com
}

Received date: Jul 10, 2020; Revised date: Jan 22, 2021; Accepted date: Jan 25, 2021

\section{Abstract}

$\mathrm{B}$ ACKGROUND: Histopathological examination is the gold standard for diagnosing meningioma and determining the treatments. However, it is invasive in nature. This study was conducted to identify intratumoral and peritumoral apparent diffusion coefficient (ADC) value and mRNA O6-methylguanine-DNA methyltransferase (MGMT) expression in meningioma.

METHODS: Data were collected from 39 patients who were clinically diagnosed with meningioma. However, only 37 patients met the inclusion criteria. These subjects then underwent examinations and received treatment from October 2017 to September 2018. Magnetic resonance imaging (MRI) data with diffusion-weighted imagingapparent diffusion coefficient (DWI-ADC) sequence, histopathological diagnosis of meningioma, and results of MGMT mRNA expression were obtained.

RESULTS: The most frequent type of low-grade and overall tumor was meningioma not otherwise specified $(56.8 \%)$.
For high-grade tumor, there were two atypical cases: atypical meningioma $(2.7 \%)$ and rhabdoid meningioma (2.7\%). Meningothelial meningioma had the highest mean value of minimum intratumoral ADC at $864.57 \pm 219$ $\mathrm{x} 10^{-3} \mathrm{~mm}^{2} / \mathrm{s}$, whereas rhabdoid meningioma had the lowest at $417 \times 10^{-3} \mathrm{~mm}^{2} / \mathrm{s}$. For minimum peritumoral ADC, rhabdoid meningioma had the highest mean value at 1,651 $\mathrm{x} 10^{-3} \mathrm{~mm}^{2} / \mathrm{s}$, while atypical meningioma has the lowest at $1,281 \times 10^{-3} \mathrm{~mm}^{2} / \mathrm{s}$. For MGMT mRNA, meningothelial meningioma had the highest mean value at $10 \pm 1.2$ fold change, whereas rhabdoid meningioma had the lowest mean at 6.18 fold change.

CONCLUSION: WHO grade I meningiomas had higher minimum intratumoral ADC values and higher MGMT mRNA expression than the high-grade tumors. Minimum peritumoral ADC values differed across the histopathological grades.

KEYWORDS: meningioma, RNA, messenger, MRI, methyltransferases, RT-PCR, ADC, MGMT mRNA

Indones Biomed J. 2021; 13(1): 97-105 


\section{Introduction}

Meningioma is a tumor that arises from arachnoid cap cells which are attached to arachnoid villi in every location. Most meningiomas are located in the skull vault and the skull base. In addition, meningiomas can be found in the spinal cord. Most cases of meningiomas are classified as World Health Organization (WHO) grade I based on histopathology examination. The prevalence of meningioma is between 50.4 and 70.7 per 100,000 population.(1) According to the statistical report from the Central Brain Tumor Registry of the United States (CBTRUS), meningioma was the most common type of brain tumor (37.1\%) in 2011-2015.(2) Meningioma is found twice as often in women than in men.(3)

Histopathological examination is still the gold standard for diagnosing brain tumor and determining subsequent treatments. However, this examination is invasive because a biopsy is needed. To obtain a preoperative diagnosis of brain tumor, radiological examination is the best modality. (4) Magnetic resonance imaging (MRI) is the modality of choice for diagnosing meningioma. With MRI, contrast differentiation and differences between intra-axial and extra-axial lesions can be shown.(3) Moreover, structural, cellular, vascular, metabolic, and functional characteristics of the tumor can be seen.(4)

To evaluate the differences between benign, atypical, and malignant meningioma, the use of conventional MRI is not sufficient.(3) Using one particular type of MRI, multiparametric MRI, the physiology and molecular properties of the brain can be shown with apparent diffusion coefficient (ADC) measurement.(5) Several studies reported that there was a decrease of ADC in high-grade tumors. A decrease in ADC value is caused by the reduction of extracellular free water diffusion and the high nuclear-tocytoplasmic ratio in high-grade tumors. As a result, the free movement of intracellular water is reduced.(3)

To determine the tumor grade, another important modality is molecular biology examination. There are numerous publications regarding the potency of molecular biomarkers as a tumor predictive factor. However, only a few of those markers are relevant in clinical settings. For instance, Co-deletion $1 \mathrm{p} / 19 \mathrm{q}$ in oligodendroglioma and O6-methylguanine-DNA methyltransferase (MGMT) promoter methylation status in glioblastoma.(6) Several studies found that in malignant tumors, MGMT expression was closely related to chemotherapy resistance.(7) MGMT gene promoter hypermethylation is a predictor of response to temozolomide (TMZ) in glioblastoma patients. One meta-analysis reported that glioblastoma patients who had MGMT promoter methylation had better survival than those who had not when they were treated using TMZ.(8) One study showed that MGMT mRNA expression played a direct role for mediating tumor sensitivity to alkylating agents. Here are three methods for detecting MGMT methylation: methylation-specific polymerase chain reaction (MSP), quantitative real-time polymerase chain reaction (PCR) or MethyLight methylation-specific quantitative real-time PCR (MethyLight qMSP), and pyrosequencing.(9) So far, eight studies regarding methylation status of MGMT promoter region have been conducted. In 6 of these 8 studies, only few meningiomas (up to $6 \%$ ) had methylated MGMT promoter. In two other studies, methylated MGMT promoter was found in $16 \%$ and 34\% meningioma cases, respectively. Another study that determined MGMT promoter methylation status in 61 meningioma cases using pyrosequencing analysis, only found that 2 out of 61 tumors (3\%) had higher mean value of methylation.(8)

In Indonesia, ADC value and MGMT mRNA measurement are not routinely done in meningioma patients in daily practice. The limitations in using ADC value are caused by the differences between results of studies regarding the efficacy of using $\mathrm{ADC}$ for determining brain tumor grade and type. Many previous studies investigating MGMT have examined its role in glial tumors. However, studies on the use of MGMT promoter methylation in meningiomas are still limited. For these reasons, this study was conducted to identify the characteristics of intratumoral ADC value, peritumoral ADC value, and MGMT mRNA expression in different types of meningioma based on the histopathological results.

\section{Methods}

\section{Study Design and Subjects Selection}

This was an observational descriptive study with crosssectional design, conducted between October 2017 and September 2018. This study was approved by the Health Research Ethics Committee Faculty of Medicine, Universitas Indonesia (No. 950/UN2.F1/ETIK/2017), the Health Research Ethics Committee Faculty of Medicine, Universitas Hasanuddin (No. 863/H4.8.4.5.31/PP36-KOMETIK/2017), the Health Research Ethics Committee RSUPN Dr. Cipto Mangunkusumo (No. LB.02.01/X.2/984/2017), and the Research Ethics Committee Dharmais Cancer Hospital (No. 091/KEPK/XI/2017). 
Thirty-nine patients were diagnosed meningioma clinically, underwent examinations, and received treatment in Cipto Mangunkusumo National General Hospital and Dharmais Cancer Hospital. However, only 37 patients met the inclusion criteria. The inclusion criteria of subjects for this study were patients diagnosed with meningioma according to histopathological results using ICD-WHO classification and had head MRI data with intratumoral and peritumoral diffusion-weighted imaging-apparent diffusion coefficient (DWI-ADC) sequences. The subjects were examined for MGMT mRNA expression using quantitative real-time polymerase chain reaction (qRT-PCR). Subjects who had incomplete examination results or incomplete medical records were excluded. All subjects had signed informed consent for participating in this study prior to examinations based on the study protocol.

\section{Histopathology Data Acquisition}

The diagnosis of meningioma was obtained from histopathological results which were categorized according to the WHO Classification of Tumors of the Central Nervous System 2016. Meningiomas were graded as WHO grade I, II, and III. The morphology codes for malignancy are based on the International Classification of Diseases for Oncology (ICD-O) [742A]. For statistical purposes, WHO grade I tumors were grouped as low-grade tumors, while WHO grade II and III tumors were grouped as high-grade tumors.(10)

\section{MRI Measurement}

All subjects underwent MRI using 1.5 Tesla Siemens Avanto. MRI images include: $\mathrm{T} 2$ weighted image $(\mathrm{T} 2 \mathrm{WI})=$ (time repetition (TR) /time echo (TE) 5160/112 ms; section thickness $5 \mathrm{~mm}$; intersection gap $1 \mathrm{~mm}$; matrix 269 x 384; field of view (FOV) $20.1 \times 23.0 \mathrm{~cm}$ ) and T1 weighted image $(\mathrm{T} 1 \mathrm{WI})=(\mathrm{TR} / \mathrm{TE} 500 / 9.4 \mathrm{~ms}$; section thickness 5 $\mathrm{mm}$; intersection gap $1 \mathrm{~mm}$; matrix $256 \times 256$; FOV 23.0 $\mathrm{x} 23.0 \mathrm{~cm}$ ). T1WI was performed in all subjects. T2 fluidattenuation inversion recovery $($ FLAIR $)=(\mathrm{TR} / \mathrm{TE} 7000 / 92$ $\mathrm{ms}$; inversion time $2214.1 \mathrm{~ms}$; section thickness $5 \mathrm{~mm}$; intersection gap $1 \mathrm{~mm}$; matrix 230 x 256; FOV 23.0 × 23.0 $\mathrm{cm}$ ). DWI was obtained in axial plane (TR/TE 4000/97; section thickness $5 \mathrm{~mm}$; intersection gap $1 \mathrm{~mm}$; matrix $128 \times 128$; FOV $23.0 \times 23.0 \mathrm{~cm}$ ). DWI was obtained with $\mathrm{b}$ values 0,500 , and $1000 \mathrm{~mm}^{2} / \mathrm{s}$. Minimum intratumoral and peritumoral $\mathrm{ADC}$ value was determined by placing the region of interest (ROI) using the workstation (Syngo MR Workplace); the placement of 5 ROI spots was done by one researcher. For DWI identification, tumor with hyperintense lesion was chosen, whereas tumor with hypointense lesion was identified for ADC measurement. ROI placement was done carefully with regard to the visual image of the tumor with an area of 1.6-1.7 $\mathrm{mm}^{2}$. After that, the lowest value for $\mathrm{ADC}$ in $\mathrm{mm}^{2} / \mathrm{s}$ was obtained.

qRT-PCR AnalysisThe qRT-PCR was done to measure MGMT mRNA gene promoter. The following primers were used : MGMT Forward: 5'GTGATTTCTTACCAGCAATTAGCA-3', MGMT Reverse: 5'- CTGCTGCAGACCACTCTGTG-3, human TATA-box binding Protein (TBP) (Forward: 5'-GAACATCATGGATCAGAACAACA-3',

The human TBP (hTBP) Reverse: 5' ATAGGGATTCCGGGAGTCAT-3'. The qRT-PCR was conducted at $95^{\circ} \mathrm{C}$ for 10 seconds and at $60^{\circ} \mathrm{C}$ for 30 seconds, in 45 cycles. RNA sample was obtained and extracted with Guanidium thiocyanate (GuSCn) (Cat \#820613, Sigma-Aldrich, Darmstadt, Germany) with Boom method RNA extraction.(11) Quantitative real-time PCR was done in triplicates using a Bio-Rad CFX Connect PCR (Biorad, Hercules, CA, USA) machine. PCR Mastermix $(22.5 \mu \mathrm{L})$ and SYBR Green QRT were mixed and prepared. The PCR results were analyzed by Bio-Rad CFX Manager 3.1 software (Biorad) using an algorithm that corrected the standard curve with material for generating is normalized gene references to hTBP.(12)

\section{Statistical Analysis}

Patients' age, gender, brain tumor diagnosis from medical records, MRI DWI-ADC results, histopathological results, and qRT-PCR results were collected. Data were analyzed descriptively using Statistical Package for the Social Sciences (SPSS) version 20 (IBM Coorporation, Armonk, NY, USA). Data included frequencies and percentages for categorical variables or mean \pm standard deviation, median, and range for numerical variables.

\section{Results}

\section{Subjects Characteristics}

From 39 subjects participated in this study, only 37 of them had complete data. Based on the selection criteria, two subjects were excluded because of incomplete examination results or incomplete medical records.

The majority of the subjects in this study were females (91.9\%) and aged 36-60 years (83.8\%) (Table 1). Low-grade tumors were more common $(94.6 \%)$ than highgrade tumors. In this study, meningioma not otherwise 
Table 1. Subjects' characteristics.

\begin{tabular}{cc}
\hline \multicolumn{1}{c}{ Characteristics } & $\begin{array}{c}\mathbf{n}(\%) \\
(\mathbf{n}=\mathbf{3 7})\end{array}$ \\
\hline Age (years old) & \\
$1-5$ & $1(2.7)$ \\
$6-10$ & $0(0)$ \\
$11-18$ & $1(2.7)$ \\
$19-35$ & $1(2.7)$ \\
$36-60$ & $31(83.8)$ \\
$61-81$ & $3(8.1)$ \\
\hline Gender & \\
Male & $3(8.1)$ \\
Female & $34(91.9)$ \\
\hline
\end{tabular}

specified accounted for the largest percentage of lowgrade and overall tumors at $56.8 \%$. There were only two high-grade tumor cases, which were atypical meningioma $(2.7 \%)$ and rhabdoid meningioma (2.7\%) (Table 2). Due to the small amount and unequal distribution of data, the statistics could not be performed with confidence interval. Lower grade meningioma tends to have a higher minimum intratumoral ADC value and mRNA expression. Minimum intratumoral $\mathrm{ADC}$ value and mRNA expression were more likely to increase in meningothelial meningioma (WHO grade I, ICD-O 9531/0) and meningioma, not otherwise specified (WHO grade I, ICD-O 9530/0) than higher grade meningiomas (atypical meningioma (WHO grade II, ICD-O 9539/1) and rhabdoid meningioma (WHO grade III, ICD-O 9538/3)).

\section{ADC value and MGMT mRNA Expression}

According to Table 2, The highest value of minimum intratumoral $\mathrm{ADC}$ was found in meningothelial meningioma $\left(864.57 \pm 219 \times 10^{-3} \mathrm{~mm}^{2} / \mathrm{s}\right)$, and the lowest minimum intratumoral ADC value was found in rhabdoid meningioma $\left(417 \times 10^{-3} \mathrm{~mm}^{2} / \mathrm{s}\right)$. For minimum peritumoral $\mathrm{ADC}$, the highest value of minimum peritumoral ADC was found in rhabdoid meningioma at $1,651 \times 10^{-3} \mathrm{~mm}^{2} / \mathrm{s}$, whereas the lowest mean value was found in atypical meningioma at $1,281 \times 10^{-3} \mathrm{~mm}^{2} / \mathrm{s}$. With regard to the highest and the lowest mean value of MGMT mRNA expression, the former was meningothelial meningioma at $10 \pm 1.2$ fold change, while the latter was rhabdoid meningioma at 6.18 fold change.

The median and range for each variable based on histopathological results were shown in Table 3. For peritumoral minimum $\mathrm{ADC}$, the data were only obtained in 19 subjects because not all tumors showed this feature, 17 of which had low-grade tumors and only 2 subjects had highgrade tumors according to histopathological results.

As shown in Figure 1 there was a case of grade I meningioma that showed noticeable edema. It was revealed that minimum intratumoral ADC value was $806 \times 10^{-3}$ $\mathrm{mm}^{2} / \mathrm{s}$ and peritumoral one was $1,333 \times 10^{-3} \mathrm{~mm}^{2} / \mathrm{s}$. In one case of atypical meningioma (WHO Grade II), there were also similar MRI findings to grade I meningioma, which were noticeable peritumoral edema and midline shifting. However, it was revealed that minimum intratumoral $\mathrm{ADC}$ quantification was lower than the grade I, which was

Table 2. Histopathological and morphological characteristics of subjects.

\begin{tabular}{|c|c|c|c|c|}
\hline \multirow[b]{2}{*}{ Variables } & \multirow[b]{2}{*}{$\begin{array}{c}\text { Frequency } \\
\text { (Percentage) } \\
{[\text { [n (\%)] }}\end{array}$} & \multicolumn{3}{|c|}{ Mean \pm SD } \\
\hline & & $\begin{array}{c}\text { Minimum } \\
\text { Intratumoral ADC } \\
{\left[\times \mathbf{1 0}^{-3} \mathrm{~mm}^{2} / \mathrm{s}\right]}\end{array}$ & $\begin{array}{c}\text { Minimum } \\
\text { Peritumoral ADC } \\
{\left[\times \mathbf{1 0}^{-3} \mathrm{~mm}^{2} / \mathrm{s}\right]}\end{array}$ & $\begin{array}{c}\text { MGMT mRNA } \\
\text { [fold change] }\end{array}$ \\
\hline \multicolumn{5}{|l|}{ Histopathological group } \\
\hline Low-grade & $35(94.6)$ & $807.45 \pm 183.00$ & $1,537.70 \pm 289.20$ & $9.55 \pm 1.33$ \\
\hline High-grade & $2(5.4)$ & $540.00 \pm 173.00$ & $1,466.00 \pm 261.60$ & $7.95 \pm 2.50$ \\
\hline \multicolumn{5}{|l|}{ Tumor grade and morphology } \\
\hline $\begin{array}{l}\text { Meningioma, not otherwise specified } \\
\text { (WHO grade I, ICD-O 9530/0) }\end{array}$ & $21(56.8)$ & $769.38 \pm 148.00$ & $1,336.00 \pm 313.00$ & $9.19 \pm 1.30$ \\
\hline $\begin{array}{l}\text { Meningothelial meningioma } \\
\text { (WHO grade I, ICD-O 9531/0) }\end{array}$ & $14(37.8)$ & $864.57 \pm 219.00$ & $1,428.25 \pm 210.00$ & $10.00 \pm 1.20$ \\
\hline $\begin{array}{l}\text { Atypical meningioma } \\
\text { (WHO grade II, ICD-O 9539/1) }\end{array}$ & $1(2.7)$ & 663.00 & 1281.00 & 9.73 \\
\hline $\begin{array}{l}\text { Rhabdoid meningioma } \\
\text { (WHO grade III, ICD-O 9538/3) }\end{array}$ & $1(2.7)$ & 417.00 & 1651.00 & 6.18 \\
\hline Total & $37(100)$ & $793.00 \pm 190.00$ & $1,369.11 \pm 282.00$ & $9.46 \pm 1.40$ \\
\hline
\end{tabular}


Table 3. Median and range value of ADC and MGMT mRNA according to histopathological results.

\begin{tabular}{lccccc}
\hline \multirow{2}{*}{ Marker } & \multicolumn{2}{c}{ Low-grade } & & \multicolumn{2}{c}{ High-grade } \\
\cline { 2 - 3 } \cline { 5 - 6 } & Median & Range & & Median & Range \\
\hline Minimum intratumoral ADC $(\mathrm{n}=37)$ & 806 & $487-1,259$ & & 540 & $417-663$ \\
Minimum peritumoral ADC $(\mathrm{n}=19)$ & 1,333 & $713-1,816$ & & 1,466 & $1,281-1,651$ \\
MGMT mRNA $(\mathrm{n}=37)$ & 9.62 & $6.15-11.62$ & & 7.95 & $6.18-9.73$ \\
\hline
\end{tabular}

Low grade : WHO Grade I; High-grade: WHO Grade II and WHO Grade III.

$663 \times 10^{-3} \mathrm{~mm}^{2} / \mathrm{s}$, whereas peritumoral one was 1,281 $\mathrm{x} 10^{-3} \mathrm{~mm}^{2} / \mathrm{s}$ (Figure 2). In a more malignant type, rhabdoid meningioma, conventional MRI finding showed more aggressive features, such as infiltrative growth pattern, signal heterogeneity, contrast enhancement, and midline shifting. Minimum intratumoral ADC value was revealed to be $417 \times 10^{-3} \mathrm{~mm}^{2} / \mathrm{s}$ according to DWI (Figure 3). These findings were confirmed with the histopathological examination. In grade I meningioma, it showed mixed type of meningothelial and transitional variants. In atypical one, it was found that mitoses index was high (5-7/10 high power field) (Figure 4).

\section{Discussion}

The gender distribution in meningioma was similar to previous studies which state that meningiomas are more frequent in women. This study found that meningioma was diagnosed before the age of 60 , especially $36-60$ age group, in contrast to previous studies.(13-15)

The MRI results showed that the higher the tumor grade, the lower the ADC value. In general, minimum intratumoral ADC was higher in low-grade tumors than highgrade ones. However, different values were found. As stated in a multicenter analysis regarding $\mathrm{ADC}$ in meningiomas to predict tumor grade and the potency of proliferation, the mean intratumoral ADC value in WHO grade I meningiomas $\left(1.05 \pm 0.39 \times 10^{-3} \mathrm{~mm}^{2} / \mathrm{s}\right)$ was significantly higher than grade II $\left(0.77 \pm 0.15 \times 10^{-3} \mathrm{~mm}^{2} / \mathrm{s}\right)$ and grade III $\left(0.79 \pm 0.21 \times 10^{-3} \mathrm{~mm}^{2} / \mathrm{s}\right)$ ones with $p=0.001$.(16) In addition, several studies stated that the ADC value of meningioma was inversely correlated with its histopathological grade. $(17,18)$ Similar results were also relatively comparable, in which the ADC mean value was significantly lower in WHO grade II meningiomas $\left(0.72 \pm 0.09 \times 10^{-3} \mathrm{~mm}^{2} / \mathrm{s}\right)$ compared with the one of WHO grade I ones $\left(1.02 \pm 0.16 \times 10^{-3} \mathrm{~mm}^{2} / \mathrm{s}\right)$ with $p<0.001$.(19) Despite these reports, a study found that
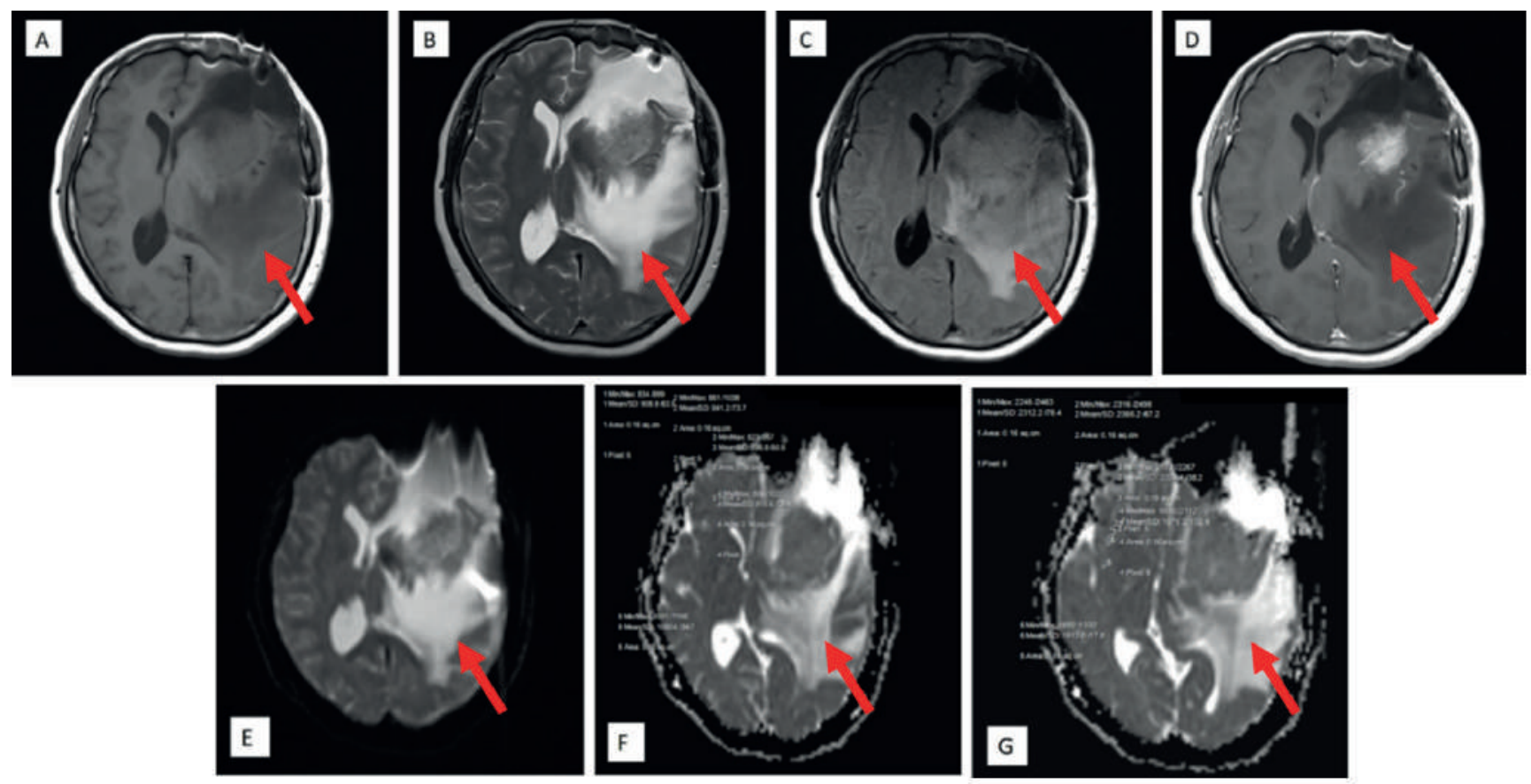

Figure 1. Meningioma, not otherwise specified case (WHO grade I, ICD 9350/0). A: T1WI; B: T2WI; C: FLAIR; D: Post-contrast T1WI; E: DWI; F: Tumoral ADC quantification; G: Peritumoral ADC quantification. Edema is shown with red arrow. 

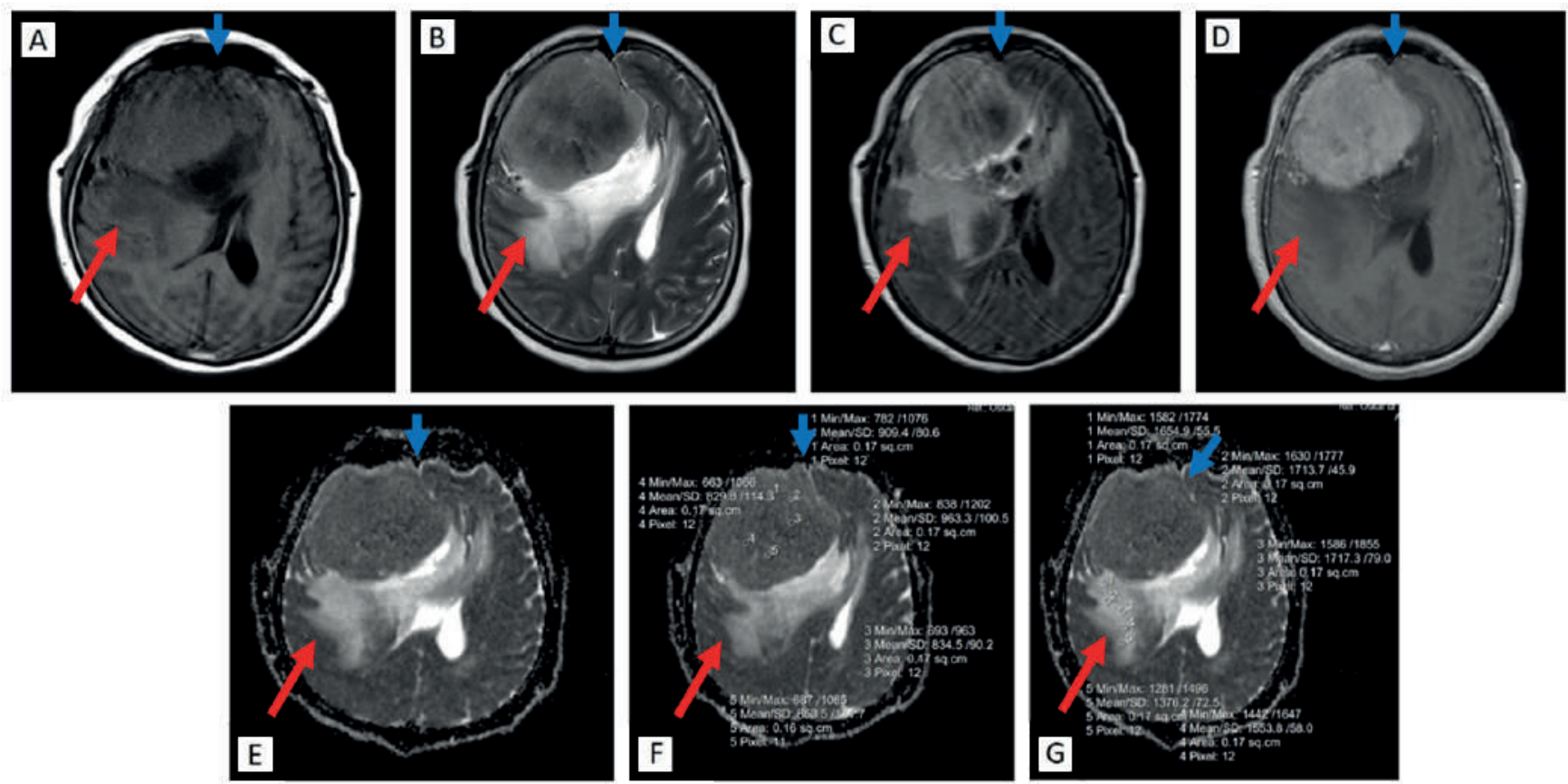

Figure 2. Atypical meningioma (WHO grade II, ICD 9359/1). A: T1WI; B: T2WI; C: FLAIR; D: Post-contrast T1WI; E: DWI; F: Tumoral ADC quantification; G: Peritumoral ADC quantification. Atypical meningioma showing peritumoral edema (red arrow) and midline shift (blue arrow).

distinguishing the histopathological grades of meningioma was not possible solely by using the ADC value.(20) Two published studies reported that DWI and ADC values were not useful in determining neither meningioma grades nor distinguishing histological subtypes. $(21,22)$
There were some cases of meningioma that did not show peritumoral edema. However, in meningioma that exhibited peritumoral edema, higher grade meningioma showed a higher minimum peritumoral ADC value. The result was slightly higher than in a previous study
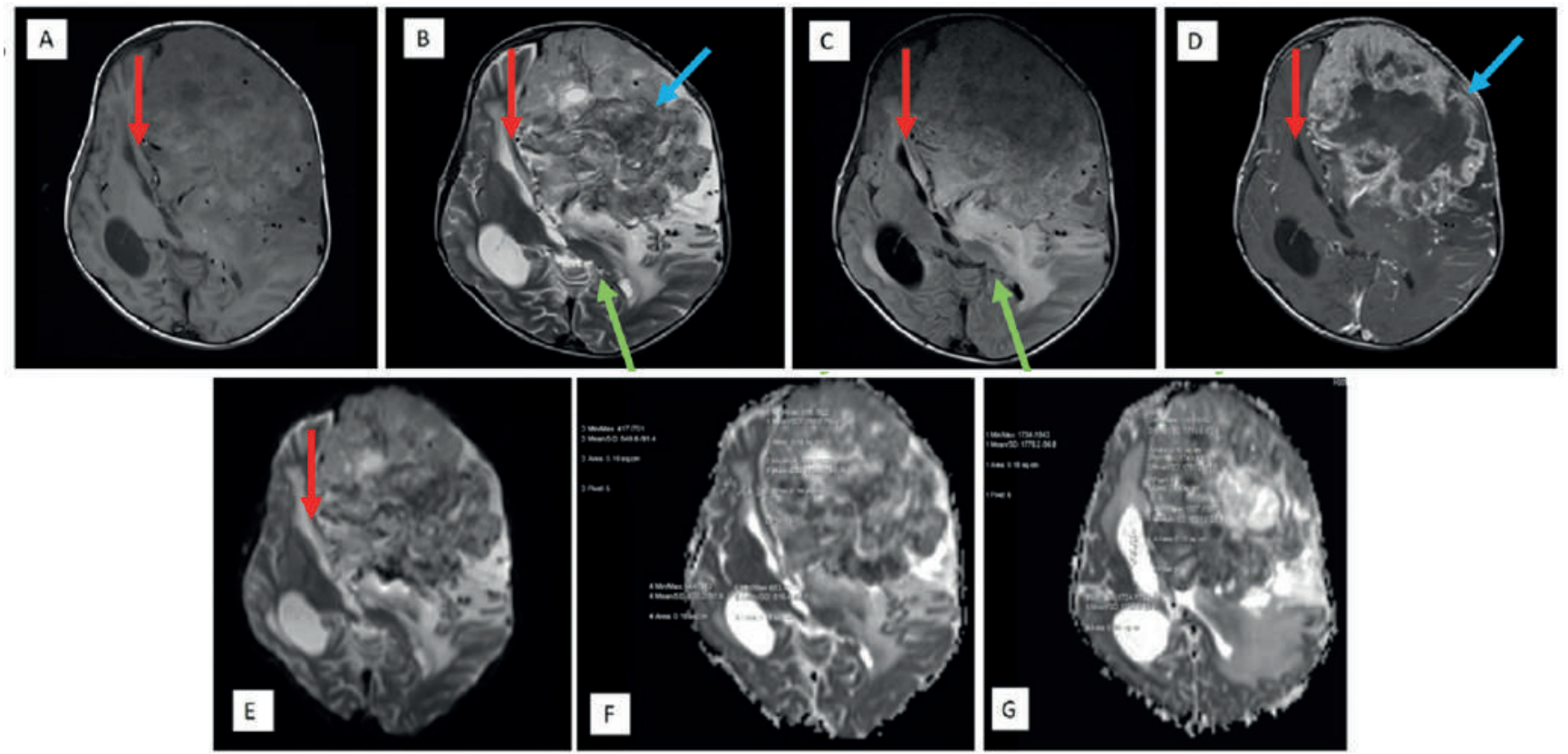

Figure 3. Rhabdoid meningioma (WHO grade III, ICD 9358/3). A: T1WI; B: T2WI; C: FLAIR; D: Post-contrast T1WI; E: DWI; F: Tumoral ADC quantification; G: Peritumoral ADC quantification. Rhabdoid meningioma shows infiltrative growth pattern (green arrow), signal heterogeneity (blue arrow), and midline shifting (red arrow) on conventional MRI. 

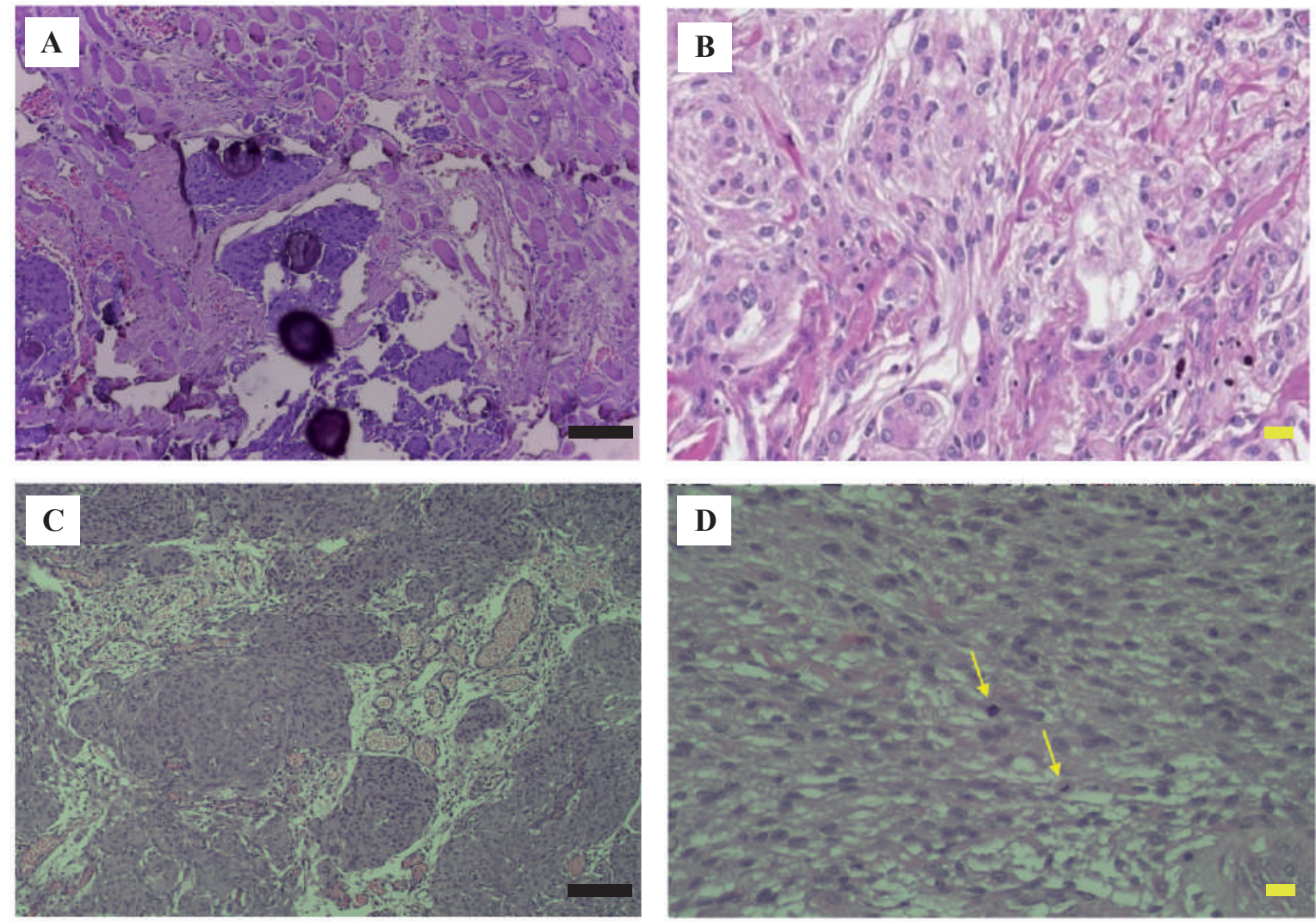

Figure 4. Histopathology of low-grade and high-grade meningioma. A and C: Mixed type transitional and meningothelial meningioma showing tumor cells in lobules and whorls with intranuclear pseudoinclusions, Black bar: $100 \mu \mathrm{m}$; B and D: Atypical meningioma showing tumor cells with increased mitotic activity (total 6/10 HPF in this case, two of them are pointed by the yellow arrows), Yellow bar: $10 \mu \mathrm{m}$.

that showed the mean value of $\mathrm{ADC}$ in high-grade meningiomas in hyperintense regions to be $1,427 \times 10^{-3}$ $\mathrm{mm}^{2} / \mathrm{s}$, while in normal-appearing white-matter regions it was $0.743 \times 10^{-3} \mathrm{~mm}^{2} / \mathrm{s}$. (23) Our results were in accordance to another published report which found that ADC, high peritumoral edema, and the absence of calcification were prognostic of advanced histopathological meningioma grade.(24)

Another variable, MGMT mRNA expression, showed that lower tumor grade tends to have higher expression. However, at the present time, there is one study regarding the expression of MGMT protein. One study found MGMT protein expression in 55\% of examined meningiomas. (25) Another study found that it was beneficial to examine MGMT protein expression status in meningeal hemangiopericytoma to predict the clinical outcome.(26) In one published research, fifteen MSP was done to examine meningiomas and found $13 \%$ of their grade I meningiomas to have their MGMT promoter methylated, and 33\% and $15 \%$ of grades II and III, respectively.(27) By utilizing the same method, similar study found $2.8 \%, 5.6 \%$, and $2.8 \%$ of 36 examined grade I, II, and III meningiomas have methylated MGMT promoter.(28) A different research used quantitative MSP (qMSP) and found $0 \%$ of their examined meningiomas exhibiting MGMT promoter methylation.(13) A recent study used pyrosequencing in 61 meningiomas and found MGMT promoter methylation in $2 \%$ of grade I, $14 \%$ of grade II, and none of grade III tumors.(8) Furthermore, several studies have demonstrated an association between methylation status and various imaging features. Additionally, methylation status was more strongly linked with brain tumor prognosis.(29-31) Relationship between MGMT promoter methylation and radiological features of MRI showed that there was no significant difference ( $p=0.14$ to $p=0.97$ ) between MGMT promoter methylation status and malignant tumor features in conventional MRI using Chi-Square test.(32)

There are several limitations to this study. First, there were only a few subjects who were enrolled. Second, the proportion of low-grade and high-grade tumors was unequal. Further study of the relationship between intratumoral ADC, peritumoral ADC, and MGMT mRNA in meningioma is needed, specifically with a larger sample size and more equal proportions of tumor grades. 


\section{Conclusion}

From our study, low grade meningiomas had higher minimum intratumoral ADC values as well as higher MGMT mRNA expression than the high-grade ones. Minimum peritumoral ADC values vary across the histopathological grades. This could be useful for identifying meningioma grades as well as its corresponding biomolecular features.

\section{Acknowledgements}

We would like to thank American Manuscript Editors for language editing service. We thank Ilhamjaya Patellongi for his advice, comments, and suggestions in statistical analysis. We also thank Vinesia Lestari Riddi for performing histopathological examination and analyzing the results.

\section{Authors Contribution}

RMdesigned the study, performed radiological examinations, conducted statistical analysis, and wrote the manuscript. AAI, BM, and JT contributed to the development of idea, study design, and supervised the study. MH performed the molecular biology experiments and supervised the findings of this study. MF performed the surgery and collected the post-surgical findings. ES performed the histopathological examination and analyzed the results. JP contributed to the development of the statistical analysis. All authors read and approved the final manuscript.

\section{References}

1. Baldi I, Engelhardt J, Bonnet C, Bauchet L, Berteaud E, Grüber A, et al. Epidemiology of meningiomas. Neurochirurgie. 2018; 64: 5-14.

2. Ostrom QT, Gittleman H, Truitt G, Boscia A, Kruchko C, BarnholtzSloan JS. CBTRUS statistical report: primary brain and other central nervous system tumors diagnosed in the United States in 2011-2015. Neuro Oncol. 2018; 20 (Suppl 4): iv1-iv86.

3. Watts J, Box G, Galvin A, Brotchie P, Trost N, Sutherland T. Magnetic resonance imaging of meningiomas: a pictorial review. Insights Imaging. 2014; 5: 113-22.

4. Villanueva-Meyer JE, Mabray MC, Cha S. Current clinical brain tumor imaging. Neurosurgery. 2017; 81: 397-415.

5. Tamrazi B, Shiroishi MS, Liu CSJ. Advanced imaging of intracranial meningiomas. Neurosurg Clin N Am. 2016; 27: 137-43.

6. Staedtke V, Dzaye OD, Holdhoff M. Actionable molecular biomarkers in primary brain tumors. Trends Cancer. 2016; 2: 338-49.

7. Ueda S, Mineta T, Nakahara Y, Okamoto H, Shiraishi T, Tabuchi $\mathrm{K}$. Induction of the DNA repair gene O6-methylguanine-DNA methyltransferase by dexamethasone in glioblastomas. J Neurosurg. 2004; 101: 659-63.

8. Panagopoulos I, Gorunova L, Leske H, Niehussman P, Johannessen LE, Staurseth J, et al. Pyrosequencing analysis of MGMT promoter methylation in meningioma. Cancer Genom Proteom. 2018; 15: 379-85.

9. Kreth S, Thon N, Eigenbrod S, Lutz J, Ledderose C, Egensperger R, et al. O6-methylguanine-DNA methyltransferase (MGMT) mRNA expression predicts outcome in malignant glioma independent of MGMT promoter methylation. PLoS One. 2011; 6: e17156. doi: 10.1371/journal.pone.0017156

10. Louis DN, Perry A, Reifenberger G, von Deimling A, FigarellaBranger D, Cavenee WK, et al. The 2016 World Health Organization classification of tumors of the central nervous system: a summary. Acta Neuropathol. 2016; 131: 803-20.

11. Tambaip T, Br Karo M, Hatta M, Dwiyanti R, Natzir R, Nasrum Mas $\mathrm{M}$, et al. Immunomodulatory effect of orally red fruit (Pandanus conoideus) extract on the expression of $\mathrm{CC}$ chemokine receptor 5 mRNA in HIV patients with antiretroviral therapy. Res J Immunol. 2018; 11: 15-21.

12. Sirait RH, Hatta M, Ramli M, Islam AA. Systemic lidocaine inhibits high-mobility group box 1 messenger ribonucleic acid expression and protein in BALB/c mice after closed fracture musculoskeletal injury. Saudi J Anesth. 2018; 12: 395-8.

13. Jabini R, Moradi A, Afsharnezhad S, Ayatollahi H, Behravan J, Raziee HR, et al. Pathodiagnostic parameters and evaluation of $\mathrm{O}$ 6 - methyl guanine methyl transferase gene promoter methylation in meningiomas. Gene. 2014; 538: 348-53.

14. Larijani L, Madjd Z, Samadikuchaksaraei A, Younespour S, Zham H, Rakhshan A, et al. Methylation of O 6-methyl guanine methyltransferase gene promoter in meningiomas - comparison between tumor grades I, II, and III. Asian Pacific J Cancer Prev. 2014; 15: 33-8

15. Van Alkemade H, De Leau M, Dieleman EMT, Kardaun JWPF, Van Os R, Vandertop WP, et al. Impaired survival and long-term neurological problems in benign meningioma. Neuro Oncol. 2012; 14: 658-66.

16. Surov A, Ginat DT, Sanverdi E, Lim CCT, Hakyemez B, Yogi A, et al. Use of diffusion weighted imaging in differentiating between malignant and benign meningiomas: a multicenter analysis. World Neurosurg. 2016; 88: 598-602

17. Watanabe Y, Yamasaki F, Kajiwara Y, Takayasu T, Nosaka R, Akiyama Y, et al. Preoperative histological grading of meningiomas using apparent diffusion coefficient at 3T MRI. Eur J Radiol. 2013; 82: 658-63.

18. Toh $\mathrm{CH}$, Castillo $\mathrm{M}$, Wong AMC, Wei $\mathrm{KC}$, Wong $\mathrm{HF}, \mathrm{Ng} \mathrm{SH}$, et al. Differentiation between classic and atypical meningiomas with use of diffusion tensor imaging. Am J Neuroradiol. 2008; 29: $1630-5$.

19. Abdel-Kerim A, Shehata M, El Sabaa B, Fadel S, Heikal A, Mazloum Y. Differentiation between benign and atypical cranial Meningiomas. Can ADC measurement help? MRI findings with histopathological correlation. Egypt J Radiol Nucl Med. 2018; 49: 172-5.

20. Lu Y, Liu L, Luan S, Xiong J, Geng D, Yin B. The diagnostic value of texture analysis in predicting WHO grades of meningiomas based on ADC maps: an attempt using decision tree and decision forest. Eur Radiol. 2019; 29: 1318-28.

21. Santelli L, Ramondo G, Della Puppa A, Ermani M, Scienza R, D'Avella D, et al. Diffusion-weighted imaging does not predict histological grading in meningiomas. Acta Neurochir (Wien). 2010; 152: $1315-9$. 
22. Sanverdi SE, Ozgen B, Oguz KK, Mut M, Dolgun A, Soylemezoglu $\mathrm{F}$, et al. Is diffusion-weighted imaging useful in grading and differentiating histopathological subtypes of meningiomas? Eur J Radiol. 2012; 81: 2389-95.

23. Provenzale JM, McGraw P, Mhatre P, Guo AC, Delong DM. Peritumoral brain regions in gliomas and meningiomas: Investigation with isotropic diffusion-weighted MR imaging and diffusion-tensor MR imaging. Radiology. 2004; 232: 451-60.

24. Hwang WL, Marciscano AE, Niemierko A, Kim DW, StemmerRachamimov AO, Curry WT, et al. Imaging and extent of surgical resection predict risk of meningioma recurrence better than WHO histopathological grade. Neuro Oncol. 2016; 18: 863-72.

25. Everson RG, Hashimoto Y, Freeman JL, Hodges TR, Huse J, Zhou $\mathrm{S}$, et al. Multiplatform profiling of meningioma provides molecular insight and prioritization of drug targets for rational clinical trial design. J Neurooncol. 2018; 139: 469-78.

26. Chang IW, Lin JW, Wu YT. The status of MGMT protein expression is a prognostic factor for meningeal hemangiopericytoma: a clinicopathologic and immunohistochemical study of 12 cases at a single institution. J Neurooncol. 2011; 105: 563-72.

27. Bello MJ, Amiñoso C, Lopez-Marin I, Arjona D, Gonzalez-Gomez $\mathrm{P}$, Alonso $\mathrm{ME}$, et al. DNA methylation of multiple promoterassociated $\mathrm{CpG}$ islands in meningiomas: relationship with the allelic status at $1 \mathrm{p}$ and 22q. Acta Neuropathol. 2004; 108: 413-21.

28. Aydemir F, Yurtcu E, Balci TB, Sahin FI, Gulsen S, Altinors N. Identification of promoter region methylation patterns of MGMT, CDKN2A, GSTP1, and THBS1 genes in intracranial meningioma patients. Genet Test Mol Biomarkers. 2012; 16: 335-40.

29. Drabycz S, Roldán G, de Robles P, Adler D, McIntyre JB, Magliocco $\mathrm{AM}$, et al. An analysis of image texture, tumor location, and MGMT promoter methylation in glioblastoma using magnetic resonance imaging. Neuroimage. 2010; 49: 1398-405.

30. Moon WJ, Choi JW, Roh HG, Lim SD, Koh YC. Imaging parameters of high grade gliomas in relation to the MGMT promoter methylation status: the $\mathrm{CT}$, diffusion tensor imaging, and perfusion MR imaging. Neuroradiology. 2012; 54: 555-63.

31. Romano A, Calabria LF, Tavanti F, Minniti G, Rossi-Espagnet MC, Coppola V, et al. Apparent diffusion coefficient obtained by magnetic resonance imaging as a prognostic marker in glioblastomas: correlation with MGMT promoter methylation status. Eur Radiol. 2013; 23: 513-20.

32. Gupta A, Omuro AMP, Shah AD, Graber JJ, Shi W, Zhang Z, et al. Continuing the search for MR imaging biomarkers for MGMT promoter methylation status: conventional and perfusion MRI revisited. Neuroradiology. 2012; 54: 641-3. 